\title{
Visual recognition: Facilitation of seeing by saying'
}

William M. Smith DARTMOUTH COLLEGE

\section{Abstract}

The vocalization of three-letter and three-syllable words was used to trigger during vocalization the visual exposure of words which were the same as the words spoken, or different. When a presented word matched the vocalized word, visual recognition was enhanced.

\section{Problem}

The general thesis that language affects perception is not new in psychology and allied fields. Moreover, it has experimental support (Miller, 1951). Knowing the names of objects and things enables one to discriminate among them better; knowing words facilitates the hearing of them. The answer to a derivative question of this general thesis is not apparent, however; that is, does the actual vocalization of language during the process of perceiving alter the manner or efficiency of perception? In the experiments reported here it is demonstrated that presenting a word visually during the act of vocalizing that word results in improved visual recognition. It remains to be shown what aspect or aspects of vocalizing is responsible for this finding.

\section{Method}

In Experiment I four different lists of 15 threesyllable words were used. The lists were equated on the basis of word frequency according to the ThorndikeLorge word count (Thorndike \& Lorge, 1944). Moreover, they were equal in terms of the representation of initial letters. Each list comprised an "uttered" list, and by altering the order of the same words, a "presented" list. The pairing across the same list in each of the four main lists was arranged so that six of the words were matched and nine were mismatched. Thus, in six instances of 15 for each of the four lists, $S$ was presented with the same word he uttered. The delay between the beginning of vocalization and the visual exposure of a word was approximately $20 \mathrm{msec}$. Vocalization of a three-syllable word typically requires $700-900 \mathrm{msec}$. Consequently, the visual exposure of a word occurred during vocalization of the first syllable of the word.

Visual recognition thresholds were determined by a modified method of limits. The words were presented in a Scientific Prototype tachistoscope on $5 \times 7$ : white cards. Each word was printed in $1 / 2$ in black block letters. The pre-exposure field with a center fixation point matched the brightness level of the word background, both being $.5 \mathrm{ft}$ lamberts. The tachistoscopic exposures were triggered by means of a voice-key through a dc comparator circuit. The latter provided the triggering pulse for the tachistoscope and was necessary to insure that once vocalization was begun the visual exposure would be continuous for the prescribed time interval. Preliminary threshold estimates were obtained

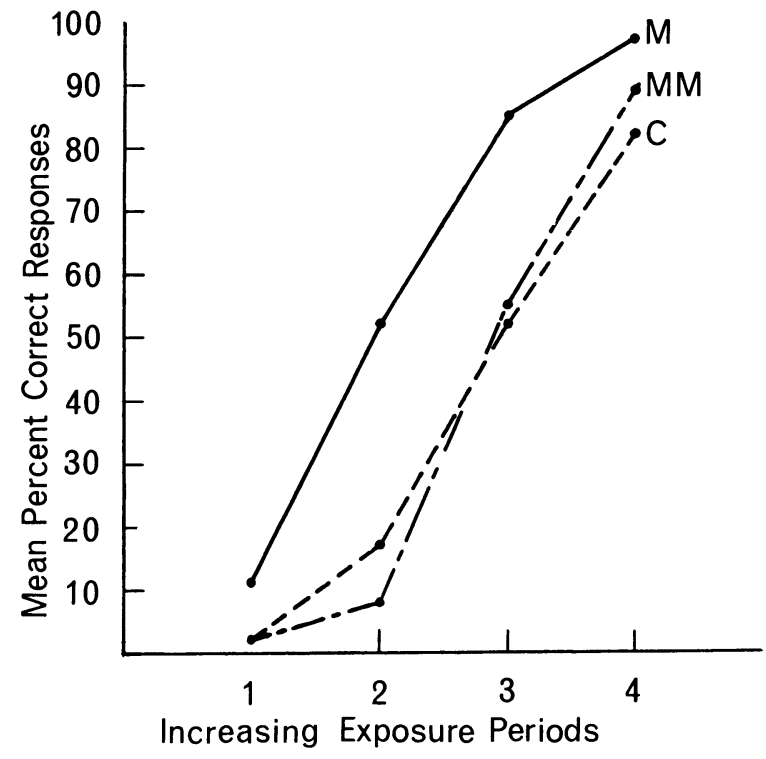

Fig. 1. Increase in visual recognition accuracy of three-syllable words with increasing exposure duration. In $\mathrm{M}$ the words presented visually matched the words spoken; in MM the words spoken were different. The control data for $\mathrm{C}$ are regular visual recognition data without vocalization. Each curve is based upon data from $12 \mathrm{Ss}$ (same Ss for M and MM).

for each $\mathrm{S}$, after which the first list was exposed at a value $5 \mathrm{msec}$. less than the estimated threshold value. At the beginning of each trial $\mathrm{S}$ was instructed to look at the fixation point, and listen for a word spoken by $\mathrm{E}$. Upon hearing the word $S$ repeated it aloud which in turn exposed the particular word for that trial. The second list of words for a given $\mathrm{S}$ was presented at a duration of $3 \mathrm{msec}$. above that used on the first list, the third list 3 msec. longer than the second, and so on. ${ }^{3}$ Each list appeared in each exposure position an equal number of times. Data were collected from $12 \mathrm{Ss}$. Control data were obtained from 12 additional Ss. The control data consisted of regular threshold measures for the same words without vocalization.

\section{Results}

The general effect of vocalization on recognition can be seen in Fig. 1. The percent correct recognition responses in each successively longer exposure period are shown for the matched words, the mismatched words, and the control words. Because of the possible tendency for $S$ to say he saw what he said the matched data for each $\mathrm{S}$ under each exposure duration was corrected by reducing the percent correct responses by the proportion of responses under the mismatched presentation in which S responded with the word he had just said.

Statistical analysis confirms what is indicated in Fig. 1. A three-way analysis of variance of the per- 


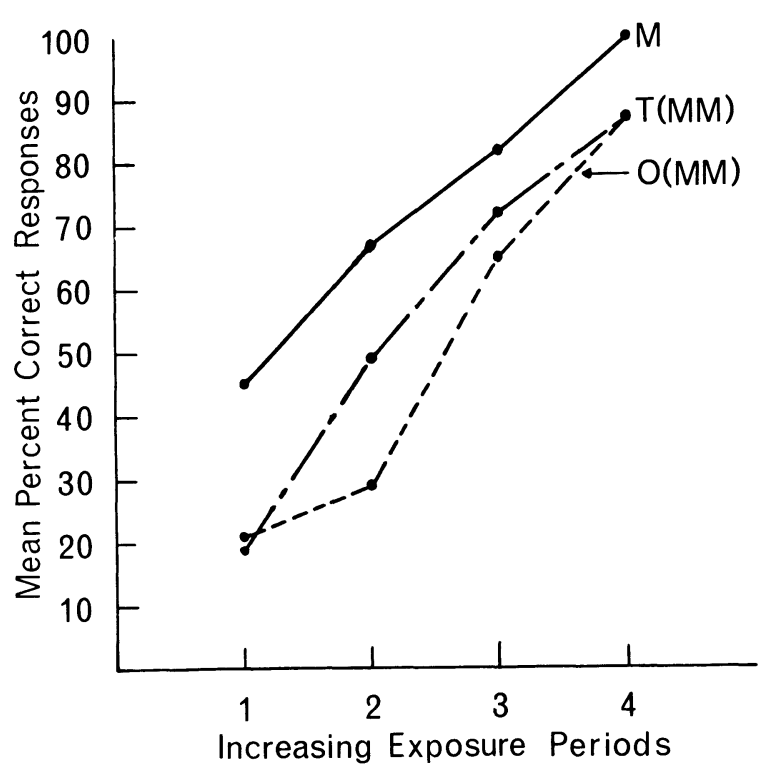

Fig. 2. Increase in visual recognition accuracy of three-letter words with increasing exposure duration. In $M$ the words presented visually matched the words spoken; in $\mathrm{T}(\mathrm{MM})$ the words presented visually were the same words used in $\mathrm{M}$, but the spoken words were different; in $O(M M)$ the mismatched words were entirely different from those used in $\mathrm{M}$ and $\mathrm{T}$. The three curves are based on data from the same $12 \mathrm{Ss}$.

centages (Finney, 1952) across the four exposure periods indicates a highly reliable difference $(p=<.001)$ between matched and mismatched data $(F=13.4$, with 1 and $47 \mathrm{df}$ ). Moreover, if the interpolated threshold values ( $50 \%$ point) in msec. is determined for each $\mathrm{S}$ and the matched-mismatched comparison is made, the mean recognition threshold is reliably smaller $(p=<.001$, one-tailed) for the matched words $(t=5.8 ; 11 \mathrm{df})$. For every $S$ the threshold for matched data was smaller.

The similarity between the control and mismatched data shown in Fig. 1 discounts the idea that the difference between the matched and mismatched conditions may have been due to an interference effect created by the mismatching of words.

In Experiment I, E read from a prepared list the word which $S$ was to vocalize on each trial. Since $E$ knew which words were matched and mismatched, it is possible that the difference between the two conditions could have been produced by unknowingly cuing S. Moreover, although balanced on the basis of frequency, the words presented visually under the matched and mismatched condition were different, and conceivably this difference could account for the data of Experiment I.

Experiment II, therefore, was carried out. In it the words spoken by $\mathrm{E}$ were put on tape beforehand by someone who did not know the matching arrangement. Moreover, three different groups of words were used within each list of four lists. In each list of 15 words five words were matched $(M)$, five were mismatched wherein the words presented visually were the same words as presented in the matched condition $(\mathrm{T})$, and, finally, five totally different mismatched words (vocalized and presented) were used (O). Four basic lists of three-letter words (with approximately equal representation of middle vowels) were randomly rearranged for each $\mathrm{S}$ and each exposure condition, subject to the three subgroupings in each list noted above. Experiment II was carried out in the same general way as Experiment I and data from 12 Ss were collected.

The results are very similar to those of Experiment I, as can be seen in Fig. 2. For each successively longer exposure period the percent correct recognitions was higher for the matched condition (M) than for both mismatched conditions ( $\mathrm{T}$ and $\mathrm{O}$ ). The matched data were correct for the possible tendency of $S$ to say what he had just said in the same way as in Experiment $\mathrm{I}$, using the data of $\mathrm{T}$ and $\mathrm{O}$ combined. By analysis of variance the $F$ value associated with the difference between $M$ and $T$ is 7.6 with 1 and $47 \mathrm{df} .(p=<.01)$. When the data are converted to recognition thresholds in msec. and t-tests are obtained, the difference between $M$ and $T$ is significant $(t=2.33 ; p=<.02$, one-tailed $)$. Also, the difference between $M$ and $O$ is significant $(t=3.58 ; p=<.005$, one-tailed). In both comparisons, the recognition thresholds for the matched condition are smaller. The difference between conditions $\mathrm{T}$-and $\mathrm{O}$ is not statistically reliable.

\section{Diseussion}

These two experiments on three-syallable and threeletter words demonstrate the facilitation of visual recognition by vocalization. The results are not sufficient in themselves, however, to identify the specific cause or causes producing the facilitation. It is possible that the observed effect is due to a factor of set, or expectancy, or to auditory or kinesthetic feedback from vocalization. The effect also might reflect the facilitative influence on one perceptual modality by parallel activity in another, that is, hearing a word while seeing it. Experiments just completed and in progress are exploring these possibilities.

\section{References}

FINNEY, D. J. Probit analysis. Cambridge: University Press, 1952. MILLER, G. A. Language and communication. New York: McGrawHill, 1951.

THORNDIKE, E. L., \& LORGE, I. The teacher's word book of 30,000 words. New York: Bureau of Publications, Columbia University, 1944.

\section{Notes}

1. This work was supported in part by National Science Foundation Grant GB-1110. I thank S. Jackson, G. T. Talbot, and R. Wise for their assistance.

2. On leave, 1964-65, California Institute of Technology and University of California, Los Angeles.

3. The absolute exposure values varied from $S$ to $S$. The values ranged from 5-23 msec. 